\title{
Vestibular apparatus: dynamic model of the semicircular canals
}

\author{
L. Gastaldi, S. Pastorelli \& M. Sorli \\ Dipartimento di Meccanica - Politecnico di Torino, Italy
}

\begin{abstract}
The vestibular apparatus plays a fundamental role in equilibrioception. It is located in the inner ear and it substantially comprises otoliths, which indicate linear acceleration, and semicircular canals, which detect rotational acceleration.

In this paper models of different complexity of the semicircular canals are reported and discussed: a monocanal hydro-elastic model and a three-canals model, in which interactions between canals are considered.

Different trends are obtained by implementing the two models in a Matlab environment. The numerical results of the monocanal model are compared with the classical torsion-pendulum model and with literature data; while, for the three-canals model, responses to angular velocity directed parallel to the physiological axes are reported.
\end{abstract}

Keywords: semicircular canals, hydroelastic model, three-canals model, mechano-transduction.

\section{Introduction}

The vestibular system is the apparatus that detects information about spatial position and movement of the head and of the body. This information is a fundamental input to control the posture, the upright position and to coordinate eye and head movement. The vestibular is quite a complex system and not all of the mechanical-nervous transduction mechanisms are completely known; this is also due to the impossibility of directly measuring human nervous signals.

Mauro et al. developed a model of the vestibular apparatus to be used for the motion cueing algorithm of a movement simulator [1], but it can be also effectively employed to better understand the functioning of the system itself or with diagnostic aims to evaluate pathologies of the balance system. 
In this paper a model of the semicircular canals, which detect only rotational movements, is presented.

Since its discovery at the end of the XIX century, the transduction biophysics of the vestibular apparatus has become an object of interest and in literature there are some models that describe the mechanics of the semicircular canals with different approximation levels: mass-spring-damping lumped parameters models $[2,3]$ that describe the macro-mechanics of the phenomenon and monocanal [4] or three-canals [5] fluidodynamic models.

The aim of the development of a new model is to obtain a rigorous mathematical description that can be used to validate simpler or linearised formulations or as a component in more complete models that account for the vestibular-ocular reflex or the mechanical-electric transactions peculiar to the semicircular canals.

Regarding the geometrical and physiological data of the semicircular canals, due to the intrinsic difficulty of a direct measurement, the authors referred to literature, considering and comparing, whenever possible, different sources and average values were taken.

\section{Physiology of the semicircular canals}

Peripheral apparatus of the vestibular system comprises different organs that perform distinct sensorial functions and perceive static and/or dynamic movements according to the six d.o.f. that characterise a body that moves in a three-dimensional space.

To register head movements, or more precisely head movement change, the vestibular apparatus comprises two different sensorial structures, both placed in the labyrinth of the inner ear:

- otoliths to detect linear acceleration;

- semicircular canals to detect angular acceleration.

Both sensorial organs contains the same receptors, the hair cells, but they are characterised by two different peculiar anatomical structures that determine the specificity in detecting linear or angular movements.

The semicircular canals (fig. 1) are three three-quarter circular ducts that intercommunicate in the utricle and they are filled with a fluid called endolymph. According to their position, canals are designated as horizontal, posterior and anterior. They are aligned approximately orthogonally to one another and the horizontal canal forms a $30^{\circ}$ angle with the physiological transverse plane, while the superior and posterior canals are aligned roughly at a $45^{\circ}$ angle to the sagittal plane. Each canal has an expanded end, the ampulla, which opens into the utricle and the superior and posterior canals join together at one extremity.

In the internal epithelium of the ampulla, next to the utricle, a gelatinous structure named the cupula is present; it introduces a discontinuity in the canal and the hair cells' cilia are imbedded inside. The cilia cells operate the transduction of a mechanical deformation due to acceleration into nervous impulses that will be elaborated by the central nervous system. 


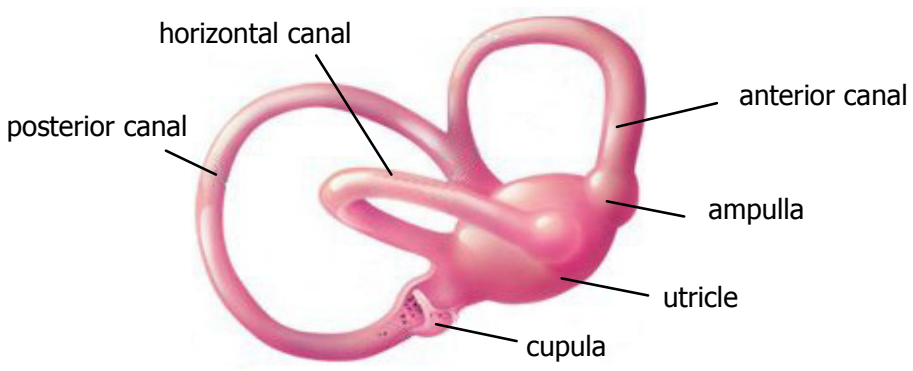

Figure 1: Physiology of the semicircular canals.

The physical principle is the following: when twisting the head, the bone structure of the semicircular canals that are integral to it accelerate with the same law, while the endolymph, due to inertial effects, moves with a lag, causing a relative displacement between the duct and the endolymphatic fluid. This flow causes a cupula deformation and a variation of the rest position of the hair cells and hence a change of the nerve impulse discharges rate carried by the vestibular nerve fibres to the brain stem.

\section{Torsion-pendulum model}

The first mathematical description of the physiology of angular motion detection is represented by the torsion-pendulum model first introduced by Steinhausen [6]. The single semicircular canal is described using a damped mass-spring model subject to an inertial force proportional to the mass. Through the years several integrations have been made to this formulation, as measurements on vestibular models pointed out that sensations detected during a rotational movement are more complex than those predicted by the torsion-pendulum model. Young and Oman [7] introduced an adaptation operator in cascade to resolve the difference between the perceived responses experimentally measured and the ones predicted by the torsion-pendulum model. Zacharias [8] suggested the introduction of a further term to also consider neural transduction dynamics.

Let us consider the canal schematisation of fig. 2 .

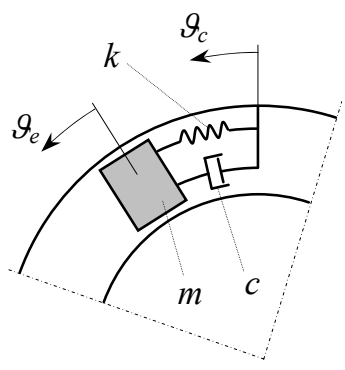

Figure 2: Torsion-pendulum model of a semicircular canal. 
The transfer function of the mechanics of the semicircular canals can be derived from the balance of the forces acting on the system, which, using the Laplace transform, may be written as:

$$
\frac{\bar{\theta}_{e}-\bar{\theta}_{c}}{\bar{\omega}}=\frac{-s}{s^{2}+\frac{c}{m} s+\frac{k}{m}}
$$

where:

$\omega$ angular velocity of the canal

$\theta_{c}$ angular displacement of the semicircular canal duct, considered integral with the head and referred to the inertial system;

$\theta_{\mathrm{e}} \quad$ angular displacement of the endolymph, referred to the inertial system;

$k \quad$ elastic constant

$c \quad$ coefficient of kinematic viscosity

$m$ mass of the endolymph;

Due to the viscous characteristics of the endolymph, this is an over-damped system and eqn. (1) becomes:

introducing:

$$
\frac{\overline{\theta_{e}}-\overline{\theta_{c}}}{\bar{\omega}} \cong \frac{-\tau_{1} \tau_{2} s}{\left(\tau_{1} s+1\right)\left(\tau_{2} s+1\right)}
$$

$$
\begin{array}{ll}
\tau_{1}=c / k & \text { long time constant } \\
\tau_{2}=m / c & \text { short time constant }
\end{array}
$$

The short time constant is dominant for high frequencies and is defined by the ratio of the mass and the viscous damping. On the contrary the long time constant, defined as the ratio of the viscous damping term and the stiffness term, influences the system behaviour for the low frequency range.

The numerical value of the short time constant can be derived by the semicircular canal physiology and there is a substantial agreement in assuming for man $\tau_{2}=0.005 \mathrm{~s}$ [9], while for the value of the long time constant data reported in bibliography are discordant. Van Egmond et al. [10], according to verbal response of humans subjected to various motion inputs, assumed a long time constant of $10 \mathrm{~s}$; Mayne [11] used the audiogyral illusion and estimated a value between 8 and $11 \mathrm{~s}$, while Groen [12], using nystagmus records, obtained $16 \mathrm{~s}$.

The estimation of the long time constant based on subjective responses is quite difficult, as it has to be purged of the effect of neural processes.

Assuming that no neural process takes place for vestibular-ocular nystagmus, Schmidt et al. [13] calculated $\tau_{1}=18 \mathrm{~s}$; as this result is consistent with the consideration of other authors $[2,12,14]$ we assumed this value to estimate the frequency response of the torsion-pendulum model.

Even if the parameters of this lumped parameter model are not easily identifiable, this mathematical description remains the most popular tool in analysing the behaviour of the semicircular canals. However, the torsionpendulum model is unable to describe the displacement of the cupula and its interaction with the endolymph. To evaluate the real displacement that describes the hair cells models, fluid mechanics are needed. 


\section{Hydroelastic-fluidodynamic monocanal model}

A detailed analysis of the fluid mechanics in a toroidal duct subjected to a pressure gradient was presented by Van Buskirk and Grant [4]. Another detailed model was obtained by Rabbitt and Damiano [5], using a perfect toroid and approximating the cupula as a linear elastic plate.

In the present study, in order to develop the monocanal model, the authors started the treatment from a straight duct with a circular section subjected to an axial motion law. Assuming that the fluid motion in the duct is laminar and that the endolymph is a newtonian and incompressible fluid, the Navier-Stokes equation for the fluid axial component is:

$$
\frac{\partial u}{\partial t}=\frac{1}{\rho}\left(-\frac{\partial P}{\partial z}+\frac{\mu}{r} \frac{\partial u}{\partial r}+\mu \frac{\partial^{2} u}{\partial r^{2}}\right)-\dot{v}
$$

where:

$\begin{array}{ll}\dot{v} & \text { duct acceleration } \\ u & \text { duct-endolymph relative velocity } \\ \rho & \text { endolymph density } \\ \mu & \text { endolymph dynamic viscosity } \\ P & \text { fluid pressure } \\ z & \text { axial coordinate } \\ r & \text { radial coordinate } \\ t & \text { time }\end{array}$

The solution of the differential equation, expressed in the Laplace dominium is:

$$
\bar{u}(r, s)=\left(\frac{1}{\rho s} \frac{\partial \bar{P}}{\partial z}+\overline{\mathrm{v}}\right)\left[\frac{J_{0}(\lambda r)}{J_{0}\left(\lambda r_{t}\right)}-1\right]
$$

where $r_{t}$ is the duct radium, $J_{0}$ is zero order Bessel's function and $\lambda^{2}=-\rho s / \mu$.

The semicircular canals can be considered, at least on first approximation, as a plane toroid; the canal and the utricle are represented with two different sections, subtended respectively by the angles $\beta$ and $\gamma$, and with internal radius $r_{c}$ and $r_{u}$, as represented in fig. $3 . R$ is the radius of the curvature of the toroid, considered constant, while $z$ is the curvilinear coordinate along the duct axis with the origin in the cupula.

As the fluid velocities in the canal are relatively low and supposing a laminar flow, forces and accelerations of the fluid that are not axially directed may be neglected. Hence we can assimilate the toroidal lengths to rectilinear ducts with a velocity equal to the peripheral velocity of the duct itself.

When considering an angular velocity $\omega$ of the semicircular canal around the axis perpendicular to the toroid and passing in the curvature centre, the velocity of the fluid relative to the canal is:

$$
\bar{u}_{c}(r, s)=\left(\frac{1}{\rho s} \frac{\partial \bar{P}_{c}}{\partial z}+\bar{\omega} R\right)\left[\frac{J_{0}(\lambda r)}{J_{0}\left(\lambda r_{c}\right)}-1\right]
$$

where $\frac{\partial \bar{P}_{c}}{\partial z}$ is the axial pressure gradient in the canal. 


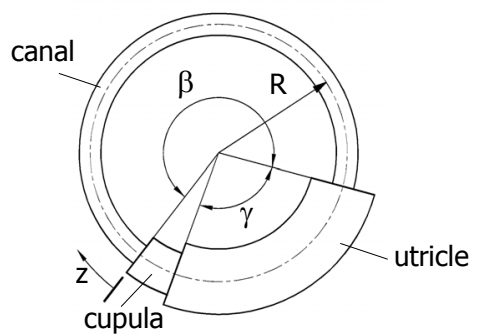

Figure 3: Discretization of the semicircular canal for the monocanal model.

In eqn. (5) the duct radius is neglected, being $\mathrm{R} \gg \mathrm{r}_{\mathrm{c}}$, and the curvilinear coordinate varies in the range $0 \leq z \leq \beta R$.

Similarly, for the utricle length, where the duct radius $r_{u}$ cannot be neglected anymore if compared to the toroid radius $R$, eqn. (4) becomes:

$$
\bar{u}_{u}(r, s)=\left(\frac{1}{\rho s} \frac{\partial \bar{P}_{u}}{\partial z}+\bar{\omega}(R+r)\right)\left[\frac{J_{0}(\lambda r)}{J_{0}\left(\lambda r_{u}\right)}-1\right]
$$

where $\frac{\partial \bar{P}_{u}}{\partial z}$ is the axial pressure gradient in the utricle and the curvilinear coordinate varies in the range $\beta R \leq z \leq(\beta+\gamma) R$.

Observing that the semicircular canal is a closed circuit, the following relation between the variation of pressure $\Delta P_{c}$ in the canal length, the variation of pressure $\Delta P_{u}$ in the utricle and the pressure difference $\Delta P_{m}$ on the cupula membrane can be written:

$$
\Delta P_{u}+\Delta P_{c}=\Delta P_{m}
$$

For the utricle, the pressure variation $\Delta P_{u}$ can be derived from eqn. (6) and, considering the conservation of flow, it can be expressed as a function of the fluid velocity relative to the canal velocity, being $A_{c} / A_{u}$ the ratio of the canal and utricle cross sections:

$$
\Delta \bar{P}_{u}=\frac{\rho s \bar{u}_{c} \frac{A_{c}}{A_{u}}-\rho s \bar{\omega}(R+r)\left[\frac{J_{0}(\lambda r)}{J_{0}\left(\lambda r_{u}\right)}-1\right]}{\left[\frac{J_{0}(\lambda r)}{J_{0}\left(\lambda r_{u}\right)}-1\right]} \cdot R \gamma
$$

To evaluate the pressure drop on the cupula, this had been modelled as an elastic circular membrane clamped at the ampullary wall along its entire perimeter and subjected to a distributed load $\Delta P_{m}$ in the axial direction. Assuming that cupula and endolymph have the same density [4, 5], the membrane deflection can be expressed in the Laplace domain as $\bar{u}_{c} / s$, representing with $h$ the membrane thickness, with $r_{m}$ the external radius of the membrane, with $E_{m}$ and $v_{m}$ Young's and Poisson's modulus, the difference of pressure $\Delta P_{m}$ is: 


$$
\Delta \bar{P}_{m}=\frac{\bar{u}_{c}}{s\left(r_{m}{ }^{2}-r^{2}\right)^{2}} \frac{16 E_{m} h^{3}}{3\left(1-v_{m}\right)}
$$

Substituting functions (8) and (9) in eqn. (7) an expression of $\Delta P_{c}$ can be derived. By introducing it in eqn. (5), the transfer function of $\bar{u}_{c} / \bar{\omega}$ can be pointed out and hence the transfer function between the angular displacement $\vartheta$ corresponding to the membrane deflection and the canal angular velocity is:

$$
\frac{\bar{\vartheta}(r, s)}{\bar{\omega}}=\frac{\bar{u}_{c}}{s R \bar{\omega}}=\frac{G s}{M s^{2}+C s+K}
$$

where coefficient $G, M, C$ and $K$ are respectively:

$$
\begin{gathered}
G=16 \mu R\left(r^{2}-r_{m}^{2}\right)^{2}[R \beta+(R+r) \gamma]\left(\frac{1}{r_{c}^{2}}-\frac{1}{r^{2}}\right)\left(\frac{1}{r_{u}^{2}}-\frac{1}{r^{2}}\right) \\
M=2 \rho R\left(r^{2}-r_{m}^{2}\right)^{2}\left[\beta\left(\frac{r^{2}}{r_{u}^{2}}-1\right)+\gamma \frac{A_{c}}{A_{u}}\left(\frac{r^{2}}{r_{c}^{2}}-1\right)\right] \\
C=8 \mu R\left(r^{2}-r_{m}^{2}\right)^{2}\left[\beta\left(\frac{r^{2}}{r_{u}^{2}}-1\right) \frac{1}{r_{c}^{2}}+\gamma \frac{A_{c}}{A_{u}}\left(\frac{r^{2}}{r_{c}^{2}}-1\right) \frac{1}{r_{u}^{2}}\right] \\
K=-\frac{256}{3} \frac{E_{m} h^{3}}{\left(1-v_{m}\right)} \frac{\mu}{\rho}\left(\frac{1}{r_{c}^{2}}-\frac{1}{r^{2}}\right)\left(\frac{1}{r_{u}^{2}}-\frac{1}{r^{2}}\right)
\end{gathered}
$$

Comparing expressions (1) and (10), it can be noticed that the transfer function of the presented hydroelastic fluidodynamic model has a structure analogous to the one derived with the more simple torsion-pendulum model, but with the advantage that the characteristic time constants $\tau_{1}=C / K, \tau_{2}=M / C$ and the gain $G / K$ can be expressed as functions of the system physiological parameters.

In fig. 4 the graphs of the frequency response $\bar{\vartheta} / \bar{\omega}$ for the torsion-pendulum model described by eqn. (2) and for the hydroelastic fluidodynamic model are shown. For the latter, the mean angular displacement of the cupula is reported. The values of the physiological parameters used for the numeric simulation and listed in tab. 1 have been extracted from literature $[4,5,15]$.

For a further comparison in fig. 4 the frequency response of the Van Buskirk and Grant [4] model is also reported.

The frequency responses are consistent, also considering the uncertainty of the physiological parameters. The perception band of the semicircular canal is in the range of $0.1 \mathrm{~Hz}$ and $100 \mathrm{~Hz}$, with an attenuation at lower and higher frequencies. In the perception band the cupula deflection and the velocity are in counter phase, while for lower and higher frequencies the system presents respectively a lag of $-90^{\circ}$ and $-270^{\circ}$. 


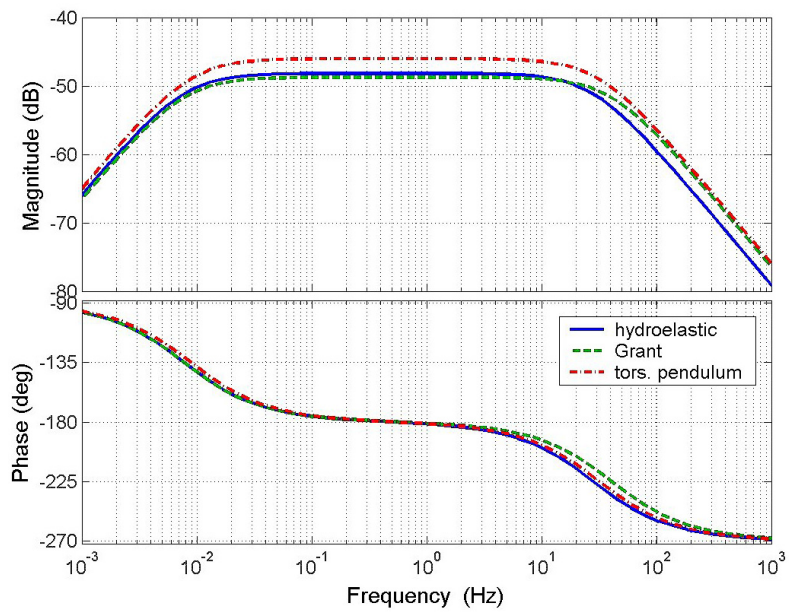

Figure 4: $\quad$ Frequency response of the mean deflection and angular velocity.

Table 1: $\quad$ Anatomical and physiological data used in the numerical models.

\begin{tabular}{|l|c|l|}
\hline canal curvature radius & $R$ & $3.175 \mathrm{~mm}$ \\
internal radius of the canal & $r_{c}$ & $0.15 \mathrm{~mm}$ \\
internal radius of the utricle & $r_{u}$ & $1.19 \mathrm{~mm}$ \\
angle subtended by the canal & $\beta$ & $1.4 \pi \mathrm{rad}$ \\
angle subtended by the utricle & $\gamma$ & $0.42 \pi \mathrm{rad}$ \\
membrane radius & $r_{m}$ & $0.9 \mathrm{~mm}$ \\
membrane thickness & $h$ & $0.57 \mathrm{~mm}$ \\
membrane Young's modulus & $E_{m}$ & $0.04 \mathrm{~N} / \mathrm{m}^{2}$ \\
membrane Poisson's modulus & $v_{m}$ & 0.5 \\
dynamic viscosity of the endolymph & $\mu$ & $10^{-3} \mathrm{~Pa} \cdot \mathrm{s}$ \\
density of the endolymph & $\rho$ & $1000 \mathrm{~kg} / \mathrm{m}^{3}$ \\
\hline
\end{tabular}

\section{Hydroelastic-fluidodynamic three-canals model}

The orientation of the three semicircular canals determines the vestibular apparatus sensibility to angular acceleration directed in any direction [16]. In general, given a stimulus, each canal answers in a different manner. To evaluate the responses it is necessary to individuate the acceleration components directed at the canals' toroids axes and then to study the system fluid-dynamic behaviour.

As depicted in fig. 5, the vertical canals, anterior and posterior, join in a common segment, with a cross section that is about twice the section of the single canal, before converging in the utricle and they came out of it at opposite ends. We want to consider, for calculation reasons, a simplified geometry that is still close to the anatomical one, analogously to what had been done for the monocanal model; the canals had been described as toroids with a circular planar section, with each being orthogonal. These planes are oriented in respect of the 
physiological axes, as shown in fig 5. The utricle is modelled as a segment of the horizontal canal with an enlarged section; the anterior and posterior canals share the vertical segment with the double section and each shares half a utricle with the horizontal canal.
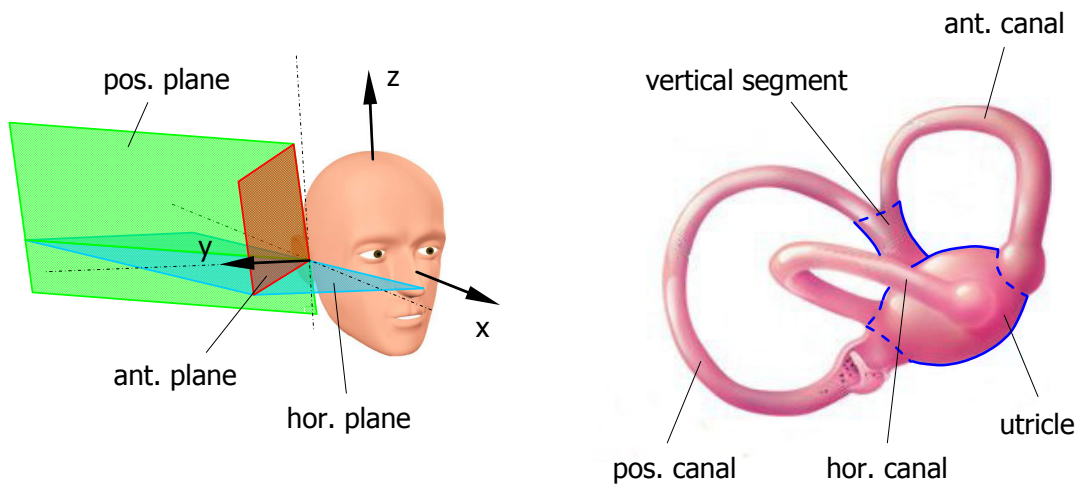

Figure 5: Discretization of the vestibular anatomical geometry.

Based on the schematisation reported in fig. 5, it is possible to write the relations between pressure in the different segments of the semicircular canals:

$$
\begin{array}{ll}
\text { horizontal canal } & \Delta P_{m / h}=\Delta P_{u}+\Delta P_{c / h} \\
\text { posterior canal } & \Delta P_{m / p}=-\Delta P_{u} / 2+\Delta P_{v}+\Delta P_{c / p} \\
\text { anterior canal } & \Delta P_{m / a}=\Delta P_{u} / 2+\Delta P_{v}+\Delta P_{c / a}
\end{array}
$$

where $\Delta P_{u}$ is the pressure variation at the utricle ends, $\Delta P_{m}$ the pressure drop on the cupula, $\Delta P_{v}$ the pressure variation in the vertical segment shared by the anterior and posterior canals and $\Delta P_{c}$ the pressure variation along the canal. Subscripts $/ h / p$ and $/ a$ indicate magnitudes relative respectively to horizontal, posterior and anterior canals.

Analogously to the monocanalar model, the endolymph is assumed to be a newtonian incompressible fluid governed by the Navier-Stokes equations. Using continuity of pressure and conservation of flow, after some algebra, the relation (14) between the membrane displacement vector of the three canals $\left[\begin{array}{lll}\theta_{h} & \theta_{p} & \theta_{a}\end{array}\right]$ and the angular velocity vector projected in the directions perpendicular to the canals planes $\left[\omega_{h} \omega_{p} \omega_{a}\right]$ becomes:

$$
\left[\begin{array}{c}
\omega_{h} \\
\omega_{p} \\
\omega_{a}
\end{array}\right]=\left[\begin{array}{lll}
A & b_{1} & c_{1} \\
a_{1} & B & c_{2} \\
a_{2} & b_{2} & C
\end{array}\right]\left[\begin{array}{c}
\vartheta_{h} \\
\vartheta_{p} \\
\vartheta_{a}
\end{array}\right]
$$

where the matrix coefficients, calculated for the cupula maximum deflexion, which is in correspondence to the canal axis, are: 


$$
\begin{aligned}
& A=\frac{\left(\beta+\frac{A_{c}}{A_{u}} \gamma\right) s^{2}+4 \frac{\mu}{\rho}\left(\frac{\beta}{r_{c}^{2}}+\frac{A_{c}}{A_{u}} \frac{\gamma}{r_{u}^{2}}\right) s+\frac{16 E_{m} h^{3}}{3\left(1-v_{m}\right) r_{m}^{4} R_{h} \rho}}{-(\beta+\gamma) s} \\
& B=\frac{2\left(\beta-\frac{A_{c}}{A_{u}} \frac{\gamma}{2}+\frac{A_{c}}{A_{v}} \eta\right) s^{2}+8 \frac{\mu}{\rho}\left(\frac{\beta}{r_{c}^{2}}-\frac{A_{c}}{A_{u}} \frac{\gamma}{2} \frac{1}{r_{u}^{2}}+\frac{A_{c}}{A_{v}} \frac{\eta}{r_{v}^{2}}\right) s+\frac{32 E_{m} h^{3}}{3\left(1-v_{m}\right) r_{m}^{4} R_{p} \rho}}{-(2 \beta-\gamma+2 \eta) s} \\
& C=\frac{2\left(\beta+\frac{A_{c}}{A_{u}} \frac{\gamma}{2}+\frac{A_{c}}{A_{v}} \eta\right) s^{2}+8 \frac{\mu}{\rho}\left(\frac{\beta}{r_{c}^{2}}+\frac{A_{c}}{A_{u}} \frac{\gamma}{2} \frac{1}{r_{u}^{2}}+\frac{A_{c}}{A_{v}} \frac{\eta}{r_{v}^{2}}\right) s+\frac{32 E_{m} h^{3}}{3\left(1-v_{m}\right) r_{m}^{4} R_{a} \rho}}{-(2 \beta+\gamma+2 \eta) s} \\
& a_{1}=\frac{A_{c}}{A_{u}} \frac{\gamma}{(2 \beta-\gamma+2 \eta)} \frac{R_{h}}{R_{p}}\left(\frac{4 \mu}{r_{u}^{2} \rho}+s\right) \\
& b_{1}=\frac{A_{c}}{A_{u}} \frac{R_{p}}{R_{h}} \frac{-\gamma}{(\beta+\gamma)}\left(\frac{4 \mu}{r_{u}^{2} \rho}+s\right) \\
& c_{1}=\frac{A_{c}}{A_{u}} \frac{R_{a}}{R_{h}} \frac{-\gamma}{(\beta+\gamma)}\left(\frac{4 \mu}{r_{u}^{2} \rho}+s\right) \\
& a_{2}=\frac{A_{c}}{A_{u}} \frac{-\gamma}{(2 \beta+\gamma+2 \eta)} \frac{R_{h}}{R_{a}}\left(\frac{4 \mu}{r_{u}^{2} \rho}+2 s\right) \\
& b_{2}=\frac{1}{(2 \beta+\gamma+2 \eta)} \frac{R_{p}}{R_{a}}\left[-\frac{A_{c}}{A_{u}} \frac{\gamma}{2}\left(\frac{1}{r_{u}^{2}}+2 s\right)-\frac{A_{c}}{A_{v}} \eta\left(\frac{1}{r_{v}^{2}}+2 s\right)\right] \\
& c_{2}=\frac{1}{(2 \beta-\gamma+2 \eta)} \frac{R_{a}}{R_{p}}\left[\frac{A_{c}}{A_{u}} \frac{\gamma}{2}\left(\frac{1}{r_{u}^{2}}+2 s\right)-\frac{A_{c}}{A_{v}} \eta\left(\frac{1}{r_{v}^{2}}+2 s\right)\right]
\end{aligned}
$$

The nomenclature used in the coefficients expressions has the same significance it had in the monocanal model, with the addition of subscripts $h p a$ and $v$ to indicate respectively the horizontal, posterior and anterior canal and the vertical shared segment; the angle $\eta$ is the arc subtended by the common vertical segment.

The frequency responses $\bar{\vartheta}_{h} / \bar{\omega}, \bar{\vartheta}_{p} / \bar{\omega}$ and $\bar{\vartheta}_{a} / \bar{\omega}$ of the cupulae mean deflection for three cases of head rotation along the physiological axes: roll (axis $x$ ), yaw (axis $y$ ) and pitch (axis $z$ ) are shown in fig. 6 .

Anthropometrical and physiological data are assumed equal for the three canals and are the same as listed in tab. 1, with the addition of $\eta=0.21 \pi \mathrm{rad}$. 

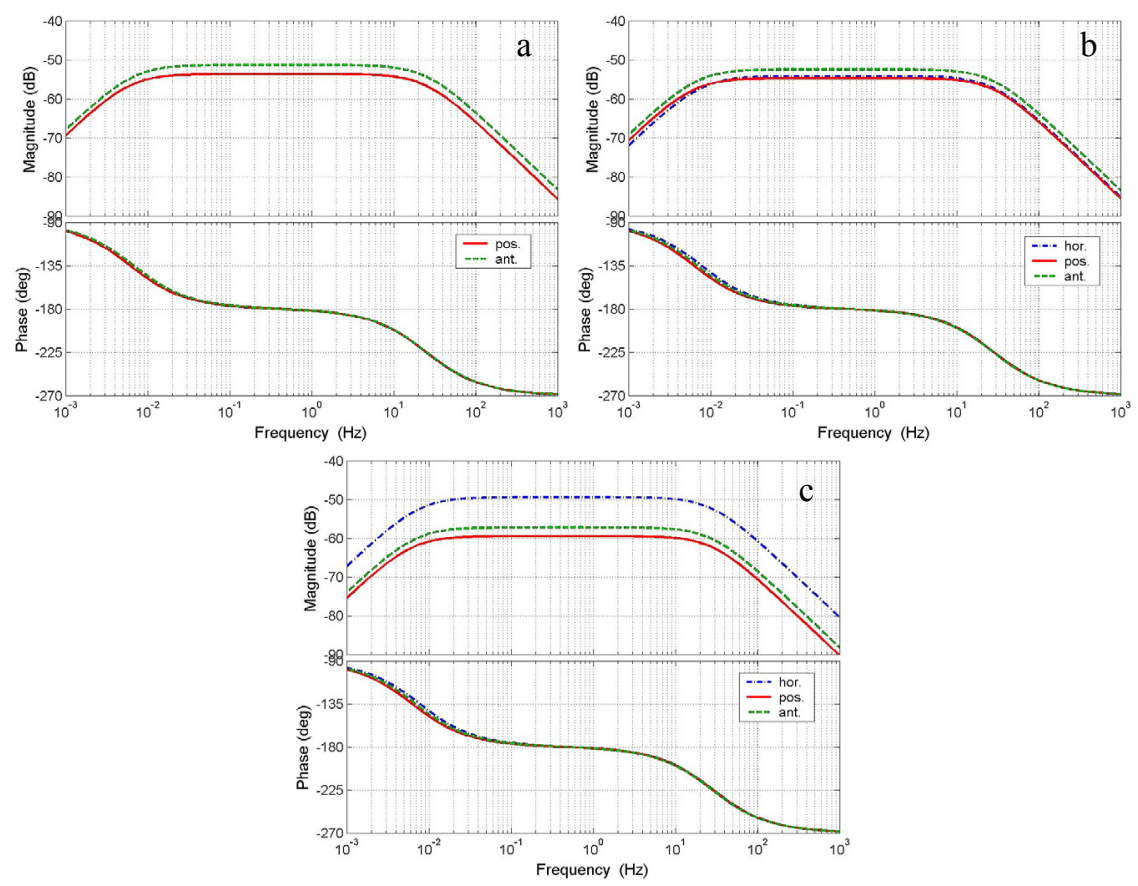

Figure 6: $\quad$ Frequency response of the three canals average cupula deflection and angular velocity for: a) roll; b) pitch and c) yaw motion.

The pass band is still the same as the monocanal model, but gain values change according to the direction of the motion. In particular the horizontal canal gain is prevalent for the yaw motion, while for the roll motion it is lower than $-100 \mathrm{~dB}$. Vertical canals present a behaviour very similar in all three motion laws tested.

\section{Conclusions}

In this paper the fluidodynamic analysis of the semicircular canals based on a simplified geometry is presented. Both a monocanal model and a three-canals model had been described. For the first model results of the frequency response between the cupula deflection and the motion law are consistent with the trends of the simpler torsional-pendulum model and with other fluidodynamic models presented in literature. For the three-canal model in literature experimental results are not present, so it would be necessary to collect some data and this is a challenging goal. In fact, direct experimental measures are not possible and for the indirect measures, exploiting the nystagmus evaluation, it is necessary to also take into account the neural answer dynamic.

The collection of experimental data is still an open question in research about the vestibular system. 


\section{References}

[1] Mauro S., Mattiazzo G., Pastorelli S., Sorli M., Development of a flight simulator: motion cueing algorithms, XVII Congresso AIMETA, Firenze, Italy, pp. 11-15, 2005.

[2] Ormsby C., Model of Human Dynamic Orientation, Ph.D. Thesis, Massachusetts Institute of Technology, Cambridge, MA, 1974

[3] Young, L.R., Dynamic control models of the semicircular canals, Dynamic Response of Biomechanical Systems, pp.133-145, 1970.

[4] Van Buskirk W. C., Grant J. W., Vestibular Mechanics, The Biomedical Engineering Handbook, Second Edition, pp. 36.1-36.14, 2000

[5] Rabbitt R. D., Damiano E. R., A Hydroelastic Model of Macromechanics in the Endolymphatic Vestibular Canal, Journal of Fluid Mechanics, 238, pp. 337-369, 1992

[6] Steinhausen W. Über die beobachtungen der cupula in der bognegangsampullen des labyrinthes des libenden hecths. Pfluegers Arch 232, pp.500-512, 1933.

[7] L. R. Young, C. M. Oman, Model for Vestibular Adaptation to Horizontal Rotation, Aerospace Medicine, 40, pp. 1076-1080, 1969

[8] Zacharias, G.L., Motion Sensation Dependence on Visual and Vestibular Cues, 1977, Ph.D. Thesis, Massachusetts Institute of Technology, Cambridge, MA.

[9] Steer, R.W., The influence of angular and linear acceleration and thermal stimulation on the human semicircular canal, Thesis, M.I.T.

[10] Van Egmond, A.A., Groen, J. J., and Jongkees, L. B. W., The Mechanics of the Semicircular Canal. Journal of Physiology. 110, pp. 1-17, 1949

[11] Mayne, R.A., A Systems Concept of the Vestibular Organs, Handbook of Sensory Physiology, Vestibular System, Springer-Verlag: New York, pp. 493-560, 1974.

[12] Groen J.J., The mechanics of the semicircular canals, J. Physiol. Lond., 110, pp.1-17, 1957

[13] Schmid, R.M., Stefanelli, M., Mira, E., Mathematical modelling. Acta Otolaryngol. 72, pp. 292-302, 1971.

[14] Goldberg, J.M., and Fernandez, C., Physiology of Peripheral Neurons Innervating Semicircular Canals of the Squirrel Monkey. II. Response to Sinusoidal Stimulation and Dynamics of Peripheral Vestibular System. Journal of Neurophysiology, 34(4): p. 661-675, 1971.

[15] Sato H., Sando I., Takahashi H., Computer-aided three-dimensional measurement of the human vestibular apparatus, Otolaryngology - Head and Neck Surgery, 107(3), pp. 405-409, 1992.

[16] Rabbitt R.D., Directional Coding of Three-Dimensional Movements by the Vestibular Semicircular Canals, Biological Cybernetics, 80, pp. 417-431, 1999. 